OPEN ACCESS

Edited by: Luca Busetto, Università degli Studi di Padova,

Reviewed by:

Marina Caputo, Università degli Studi del Piemonte

Orientale, Italy

Kok Yong Chin, National University of Malaysia, Malaysia

*Correspondence: Qian Chen chenqian01@xinhuamed.com.cn

${ }^{\dagger}$ These authors have contributed equally to this work and share first authorship

Specialty section: This article was submitted to Obesity,

a section of the journa

Frontiers in Endocrinology

Received: 17 November 2021 Accepted: 29 December 2021

Published: 17 February 2022

Citation:

Tang W, Zhan W, Wei M and Chen Q (2022) Associations Between Different

Dietary Vitamins and the Risk of Obesity in Children and Adolescents: A Machine Learning Approach. Front. Endocrinol. 12:816975 doi: 10.3389/fendo.2021.816975

\section{Associations Between Different Dietary Vitamins and the Risk of Obesity in Children and Adolescents: A Machine Learning Approach}

\author{
Weifeng Tang ${ }^{1 \dagger}$, Wenqiang Zhan ${ }^{1,2 \dagger}$, Mengdan Wei ${ }^{1}$ and Qian Chen ${ }^{1 *}$ \\ ${ }^{1}$ Ministry of Education-Shanghai Key Laboratory of Children's Environmental Health, Xinhua Hospital, Shanghai Jiao Tong \\ University School of Medicine, Shanghai, China, ${ }^{2}$ School of Public Health, Shanghai Jiao Tong University School of \\ Medicine, Shanghai, China
}

Backgrounds: Simultaneous dietary intake of vitamins is considered as a common and real scenario in daily life. However, limited prospective studies have evaluated the association between multivitamins intake and obesity in children and adolescents.

Objectives: This study aimed to evaluate the relationship between the intake of different dietary vitamins and the risk of obesity in children (6-11 years) and adolescents (12-19 years).

Methods: We conducted a cross-sectional study based on data from U.S. National Health and Nutrition Examination Survey, 2013-2016. A total of 3634 children and adolescents were included who had available data on dietary vitamins, obesity and covariates. We analyzed the dietary intake levels of nine vitamins, including vitamin $A$, vitamin $B_{1}$, vitamin $B_{2}$, vitamin $B_{6}$, vitamin $B_{12}$, vitamin $C$, vitamin $D$, vitamin $E$, vitamin $K$. Multivariate logistic regression was used to model the associations between vitamins and obesity. Bayesian kernel machine regression (BKMR) was employed to explore the joint and independent effects of vitamins on obesity among children and adolescents.

Results: In the multivariate logistic regression model, five vitamins (vitamin $A$, vitamin $B_{1}$, vitamin $B_{2}$, vitamin $B_{12}$, and vitamin $D$ ) were negatively associated with obesity in children and adolescents. BKMR analysis showed that when the concentration of the nine vitamins was at or above the 55th percentile compared with the median value, the combined intake of these vitamins could significantly reduce the risk of obesity in children and adolescents. Potential interactions between vitamin $\mathrm{B}_{2}$ and vitamin $\mathrm{B}_{12}$ in increasing the risk of obesity in children and adolescents were observed.

Conclusions: We determine the combined effects of multivitamins on obesity in children and adolescents, and observe a significant interaction between vitamin $\mathrm{B}_{2}$ and vitamin $\mathrm{B}_{12}$. Further cohort studies are needed to clarify the health effects of multivitamins intake in a larger population.

Keywords: multivitamins, children, adolescents, obesity, Bayesian Kernel machine regressioN, diet 


\section{INTRODUCTION}

Obesity is a complex disease, which is intertwined with biological, developmental, environmental, behavioral and genetic factors (1). Given that the obesity rate of children and adolescents worldwide is rising, it is necessary to pay special attention to the obesity in children and adolescents (2). Childhood obesity is a continuing and serious international health problem affecting approximately $17 \%$ of American children and adolescents, and will threaten their health and longevity in adulthood (3). However, the reasons for the large differences in the prevalence of obesity among children are still unclear.

Childhood is identified as the golden age of investing in obesity prevention. Considering the multi-factor origin of obesity, a better understanding of the influencing factors is essential for effective treatment and prevention $(4,5)$. Micronutrient status may be a significant factor for the development of obesity during childhood (6). At the same time, due to the high correlation between dietary intake of vitamins, studies had shown that there was a negative correlation between vitamin $A$, vitamin $B_{12}$ and folic acid status and the intake of thiamine and riboflavin $(7,8)$. Therefore, it is urgent to examine the significant effects of common multivitamins in children and adolescents on obesity. Most vitamins are deficient in obese individuals, especially water-soluble vitamins, such as vitamin $B_{12}$ and vitamin $C$. However, in the case of obesity, multivitamins are less evaluated. Adipose tissue is considered to be a metabolic and endocrine organ (9). Excessive intakes of vitamins can interfere with body homeostasis, but vitamins deficiency can exacerbate pathological conditions (10). Therefore, there is a great need to assess the relationship between vitamins status and obesity in children and adolescent.

However, the majority of the current nutritional epidemiological studies only evaluated the impact of individual vitamin intake on childhood obesity (11-14). Those studies only focused on the association between vitamins in isolation and childhood obesity, rather than considering all vitamins intakes, which might have additive, synergistic, antagonistic, or enhancing effects when they took together. In view of the urgent need for new methods, the development of multiple exposure model statistical methods has recently received considerable attention (15). However, few studies had taken multivitamins as a whole and used advanced statistical methods to examine vitamin mixtures (16). Bayesian Kernel Machine Regression (BKMR) is a novel statistical method that solves interactions and nonlinear relationships by flexibly modeling exposures, and has been widely used in many different medical research fields $(17,18)$.

Therefore, this study aims to explore the association between 9 vitamins and obesity in children and adolescents aged 6 to 19 in the U.S. National Health and Nutrition Examination Survey, including vitamin $A$, vitamin $B_{1}$, vitamin $B_{2}$, vitamin $B_{6}$, vitamin $B_{12}$, vitamin $C$, vitamin $D$, vitamin $E$, vitamin $K$. Given the effect of multivitamins intake, we use BKMR model to examine the joint effect of multivitamins on obesity in children and adolescents and assess the interaction between vitamins.

\section{METHODS}

\section{Study Participants}

The current study used cross-sectional data from National Health and Nutrition Examination Survey (NHANES) 20132016. NHANES is a survey designed to assess the health and nutrition of a nationally representative sample of the noninstitutionalized U.S. population. A detailed description of the study design has been previously published (19). The survey is maintained and managed by the National Bureau of Statistics of the Center for Health Statistics (NCHS) and the Centers for Disease Control and Prevention (CDC). Participants aged 6-19 years with complete dietary intake data were included in the study population. Participants with unreliable diet data, missing vitamin data, or potential confounding factors were further excluded from the analysis, and a total of 3634 subjects were included in the analysis. The detailed flow chart of this study was shown in Figure 1. All participants provided written informed consent and the Institutional Review Board of the NCHS approved the survey protocol (20).

\section{Exposure and Outcome Variables}

NHANES used 24-hour dietary recalls (24HRs) to obtain dietary information. For each participant, daily intake of nutrients from food/beverages and dietary supplements was calculated using the US Department of Agriculture's Diet and Nutrition Database for dietary research and NHANES dietary supplement calculations, respectively. The intake level of nine common vitamins (vitamin $A$, vitamin $B_{1}$, vitamin $B_{2}$, vitamin $B_{6}$, vitamin $B_{12}$, vitamin $C$, vitamin $D$, vitamin $E$, vitamin $K$ ) of each participant was assessed by the food frequency questionnaire. During the health check in the mobile check center, the weight and height were collected by well-trained health technicians. Body mass index (BMI) category was calculated for children and adolescents aged 6-19 years in NHANES and defined as four levels: underweight (BMI $<5$ th percentile), normal weight (5th $\leq$ BMI $<85$ th percentile), overweight (85th $\leq$ BMI $<$ 95th percentile) and obese $(B M I \geq$ 95th percentile) (21).

\section{Covariates}

Demographic characteristics were obtained through questionnaire surveys, including age (years), ratio of family income to poverty (PIR), energy, and serum cotinine concentration as continuous variables. Education level was grouped into high school and above, high school, elementary school and below. The race was divided into Mexican American, Other Hispanic, Non-Hispanic White, Non-Hispanic Black, Other/multi-racial (22).

According to National Cholesterol Education Program guidelines (23), abnormal serum TC, serum HDL-C, serum LDL-C, fasting TAG and fasting plasma glucose concentrations, and abnormal HOMA-IR score, were defined as follows: TC $\geq 200$ $\mathrm{mg} / \mathrm{dl}, \mathrm{HDL}-\mathrm{C} \leq 35 \mathrm{mg} / \mathrm{dl}, \mathrm{LDL}-\mathrm{C} \geq 130 \mathrm{mg} / \mathrm{dl}, \mathrm{TAG} \geq 150 \mathrm{mg} / \mathrm{dl}$, glucose $\geq 100 \mathrm{mg} / \mathrm{dl}$ and HOMA-IR $\geq 4.39$ (24).

\section{Statistical Analysis}

Continuous variables were expressed as mean \pm standard deviation or median [interquartile range (IQR)], and the 


\section{Participants included in the NHANES (2013-2016)}

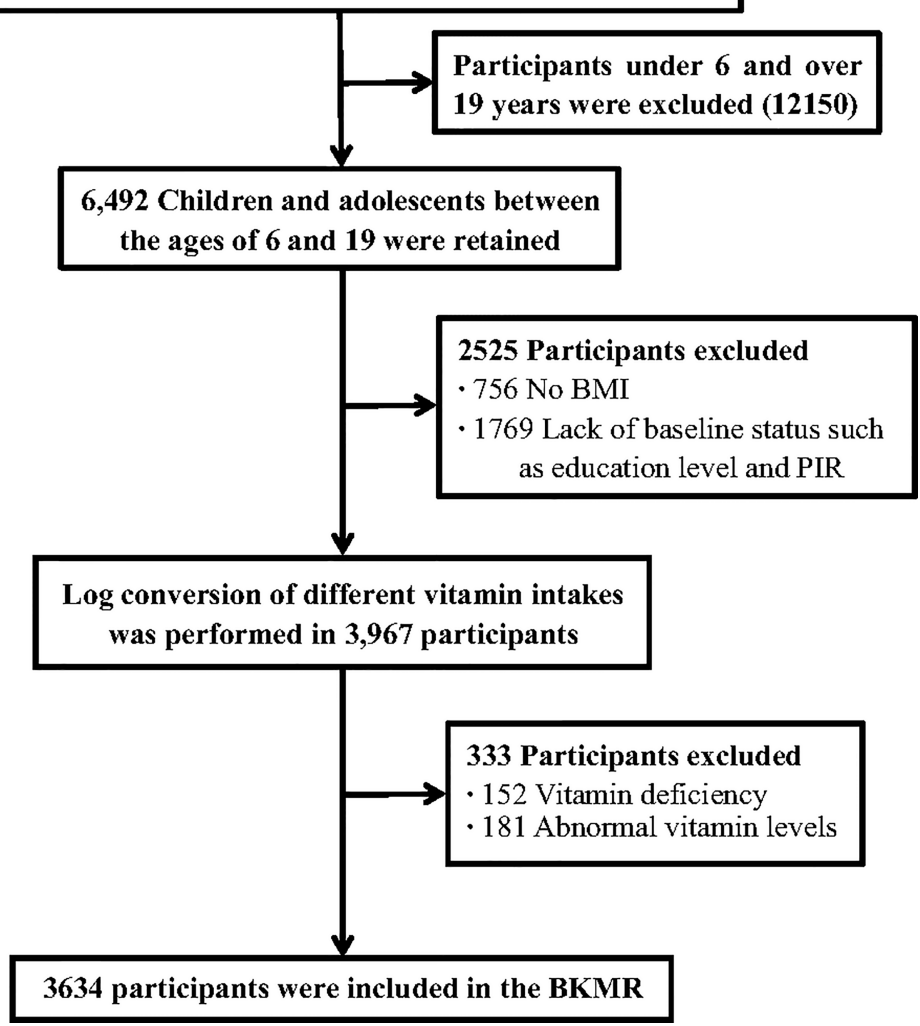

FIGURE 1 | Selection process of subjects. BKMR, Bayesian kernel machine regression.

number of categorical variables (percentage) was expressed. Spearman correlation was used to evaluate the association between dietary vitamin intake and obesity in children and adolescents. Given that the distributions of vitamins were skewed to the right, we performed a logarithmic transformation. In addition, we used multivariate linear regression models to evaluate the association between individual log-transformed vitamin intakes and obesity in children and adolescents.

Restricted cubic splines and correlation coefficient matrix plots were used to evaluate the non-linearity of multivitamins intake on obesity in children and adolescents and the interaction between vitamins. The BKMR analysis was performed to evaluate the combined effect of multi-vitamins based on Gaussian process regression. The BKMR method uses a Bayesian variable selection process to construct an exposure-response function and infer highly correlated vitamins. First, we analyzed the cumulative effect on obesity by fixing all nine vitamins to a specific percentile and increasing the 5 th percentile. Then, while keeping the other eight vitamins at the median, we used the exposure-response function to explore the association between a single vitamin and obesity. Finally, the predicted response function of a single vitamin to other vitamins at different quantiles, and the rest of the vitamins fixed at the median was studied. The group posterior inclusion probability (groupPIP) and the conditional posterior inclusion probability (condPIP) were calculated, representing the probability of a mixture group and a particular vitamin within the group, respectively. In BKMR model, exposure variables with large PIP values may be important for the overall impact of obesity risk (25). BKMR model was fit 50,000 iterations using the Markov Chain Monte Carlo (MCMC) sampler.

Sensitivity analysis evaluates the robustness of the results by analyzing the correlation between dietary vitamin intake and metabolic parameters (including serum TC, serum HDL-C, serum LDL-C, fasting TAG and fasting plasma glucose concentrations, and HOMA-IR score).

The analysis was performed on the $\mathrm{R}$ software (version 3.6.0; $\mathrm{R}$ Core Team), and the statistical significance level on both sides was set to $\alpha=0.05$.

\section{RESULTS}

Our analysis included 3634 children and adolescents aged 6-19 from NHANES (2013- 2016). The median (IQR) age was 12 (916) years old, and the BMI was $20.80(17.60-24.83) \mathrm{kg} / \mathrm{m}^{2}$ (Table 1). Among them, 533 children and adolescents were obese $(14.7 \%)$. The majority of participants were non-Hispanic 
TABLE 1 | Baseline characteristics among 3634 participants in the NHANES 2013-2016.

\begin{tabular}{|c|c|}
\hline Characteristic & Frequency $(\%)$ or Median (IQR) \\
\hline Age (years) & $12(9-16)$ \\
\hline \multicolumn{2}{|l|}{ Gender } \\
\hline Female & 1776 (48.9\%) \\
\hline Male & $1858(51.1 \%)$ \\
\hline Body mass index $\left(\mathrm{kg} / \mathrm{m}^{2}\right)$ & $20.80(17.60-24.83)$ \\
\hline \multicolumn{2}{|l|}{ Education level } \\
\hline Primary school and below & $1920(52.8 \%)$ \\
\hline Middle school & $855(23.5 \%)$ \\
\hline Senior high school and above & $856(23.6 \%)$ \\
\hline \multicolumn{2}{|l|}{ Race/ethnicity } \\
\hline Mexican American & 845 (23.3\%) \\
\hline Other Hispanic & $413(11.4 \%)$ \\
\hline Non-Hispanic White & $1016(28.0 \%)$ \\
\hline Non-Hispanic Black & $829(22.8 \%)$ \\
\hline Other/multi-racial & $531(14.6 \%)$ \\
\hline Poverty-income ratio & $1.29(0.77-2.94)$ \\
\hline Serum cotinine (ng/mL) & $0.03(0.01-0.17)$ \\
\hline Daily energy intake (kcal/day) & $1846(1397-2392)$ \\
\hline Vitamin A (mcg) & 475 (264- 765) \\
\hline Vitamin $\mathbf{B}_{1}(\mathrm{mg})$ & $1.44(1.00-2.02)$ \\
\hline Vitamin $B_{2}(\mathrm{mg})$ & $1.74(1.17-2.45)$ \\
\hline Vitamin $\mathbf{B}_{6}(\mathrm{mg})$ & $1.54(1.02-2.25)$ \\
\hline Vitamin $\mathbf{B}_{12}(\mathrm{mcg})$ & $3.95(2.23-6.27)$ \\
\hline Vitamin C (mg) & 47.60 (18.40- 101.03) \\
\hline Vitamin D (mcg) & $3.90(1.60-7.10)$ \\
\hline Vitamin E (mg) & $6.30(4.11-9.43)$ \\
\hline Vitamin K (mcg) & $48.20(28.80-84.13)$ \\
\hline $\mathrm{TC} \geq 200 \mathrm{mg} / \mathrm{dl}$ & $923(25.3 \%)$ \\
\hline HDL-C $\leq 35 \mathrm{mg} / \mathrm{dl}$ & $318(8.7 \%)$ \\
\hline LDL-C $\leq 130 \mathrm{mg} / \mathrm{dl}$ & $774(21.2 \%)$ \\
\hline Fasting TAG $\geq 150 \mathrm{mg} / \mathrm{dl}$ & $691(19.0 \%)$ \\
\hline Fasting glucose $\geq 100 \mathrm{mg} / \mathrm{dl}$ & 1506 (41.4\%) \\
\hline HOMA-IR score $\geq 4.39$ & 797 (21.9\%) \\
\hline
\end{tabular}

TC, total cholesterol; HDL-C, HDL-cholesterol: TAG, Triacylglyceride; LDL-C, LDL cholesterol; HOMA-IR, homeostasis model assessment of insulin resistance.

white $(28 \%)$ and had an elementary school or lower education (52.8\%). Their serum cotinine and daily energy intake were 0.03 (0.01-0.17) ng/mL and 1846 (1397-2392) kcal/d, respectively. The intakes of various vitamins were also presented in Table $\mathbf{1}$.

In the multivariate logistic regression model (Table 2), we found that vitamin $A$, vitamin $B_{1}$, vitamin $B_{2}$, vitamin $B_{12}$, and vitamin $\mathrm{D}$ were negatively associated with obesity in children and adolescents. The results of restricted cube plots and correlation coefficient matrix plots showed that multivitamins and obesity risk presented a non-linear correlation and there was a high correlation between vitamins (Supplementary Figures 1, 2).

Moreover, we used the BKMR model to evaluate the interaction between dietary intake of vitamins and obesity in children and adolescents. The estimated PIP of the BKMR model of vitamins and obesity risk in children and adolescents was shown in Table 3. The PIP of the three groups was higher than 0.5 , and the condPIP of vitamin $\mathrm{B}_{12}(0.88)$ and vitamin $\mathrm{D}(0.65)$ were the highest in their groups.

At the same time, in order to investigate the potential nonlinearity of the exposure response function, the estimated univariate exposure-response function of each vitamin was demonstrated graphically in Figure 2. We found that the direction of exposure responses via the BKMR model was
TABLE 2 | The association between each vitamin and body mass index in 3634 participants based on logistic regression model.

\begin{tabular}{lcc}
\hline Vitamins & OR (95\% Cl) & P-value* \\
\hline Vitamin E & $0.96(0.62-1.49)$ & 0.853 \\
Vitamin A & $0.80(0.63-0.99)$ & 0.036 \\
Vitamin $B_{1}$ & $0.67(0.41-0.84)$ & 0.023 \\
Vitamin $B_{2}$ & $0.72(0.48-0.93)$ & 0.026 \\
Vitamin $B_{6}$ & $0.90(0.61-1.34)$ & 0.608 \\
Vitamin $B_{12}$ & $0.61(0.39-0.81)$ & 0.012 \\
Vitamin C & $0.98(0.82-1.16)$ & 0.770 \\
Vitamin D & $0.86(0.60-0.96)$ & 0.042 \\
Vitamin K & $0.84(0.63-1.11)$ & 0.211
\end{tabular}

${ }^{*}$ All models were adjusted for age, gender, race, education level, daily energy intake, serum cotinine, and poverty-income ratio.

TABLE 3 | PIPs for group inclusion and conditional inclusion into obesity, using BKMR model.

\begin{tabular}{lccc}
\hline Vitamin & Group & groupPIP & conPIP \\
\hline Vitamin A & 1 & 1 & 1 \\
Vitamin E & 4 & 0.32 & 0.16 \\
Vitamin C & 4 & 0.32 & 0.25 \\
Vitamin D & 4 & 0.32 & 0.41 \\
Vitamin K & 4 & 0.32 & 0.18 \\
Vitamin $B_{1}$ & 3 & 0.76 & 0.12 \\
Vitamin $B_{2}$ & 2 & 0.62 & 0.35 \\
Vitamin $B_{12}$ & 3 & 0.76 & 0.88 \\
Vitamin D & 2 & 0.62 & 0.65
\end{tabular}

PIPs, posterior inclusion probabilities; BKMR, Bayesian kernel machine regression. Model were adjusted for age, sex, education level, race/ethnicity, poverty-income ratio, serum cotinine and daily energy intake.

generally consistent with the associations observed in the single-vitamin multivariate logistic model. When all the other vitamins were fixed at their median concentrations, vitamin $A$, vitamin $D$, vitamin $B_{1}$, vitamin $B_{2}$ and vitamin $B_{12}$ were positively correlated with high-density lipoprotein (HDL). The change in the content of the five vitamins from $25 \%$ to $75 \%$ was associated with a significant reduction in the risk of obesity.

The overall associations between the vitamins mixture and obesity were shown in Figure 3A. The risk of obesity increased significantly with increasing total vitamins mixture levels. In order to assess the potential nonlinearity of the exposureresponse function, we then estimated the univariate relationship between each vitamin in the pro-inflammatory nutrient group and the risk of obesity, with all remaining nutrients fixed at the 50th percentile. The risk of childhood obesity increased significantly with increased concentration levels of vitamin $A$, vitamin $D$, vitamin $B_{1}$, vitamin $B_{2}$, and vitamin $B_{12}$ (Figure $3 B$ ). The Figure 4 showed the interaction between vitamin $B_{2}$ and vitamin $B_{12}$ that may increase the risk of obesity in children and adolescents. When the other seven vitamins were at the median value, the positive slope of vitamin $B_{12}$ became steeper at higher levels of vitamin $B_{2}$.

The results of the sensitivity analysis showed that the associations between the nine dietary vitamins and metabolic parameters were consistent with the main results (Supplementary Table 1). 


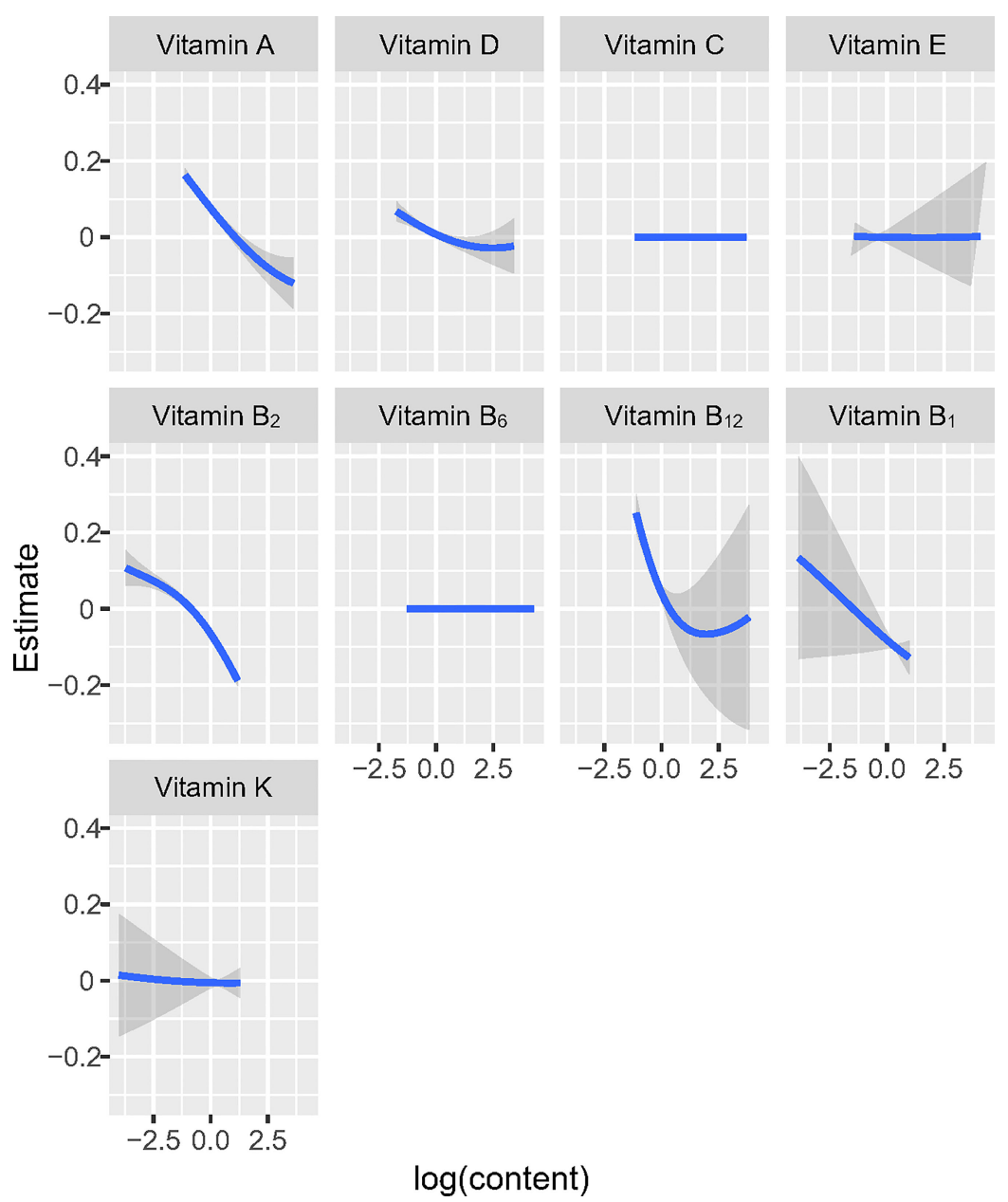

FIGURE 2 | Univariate exposure-response functions and 95\% confidence interval for association between the intake of single vitamin when the intake of other vitamins is fixed at the median.

\section{DISCUSSION}

In this study, we found that the increase of five vitamins (vitamin $A$, vitamin $B_{1}$, vitamin $B_{2}$, vitamin $B_{12}$, and vitamin $D$ ) was significantly negatively correlated with the occurrence of obesity in children and adolescents. The BKMR analysis showed that there was an interaction between vitamin $B_{2}$ and vitamin $B_{12}$. When the vitamin intake was higher than the 55th percentile, the mixed intake of the five vitamins had a negative effect on obesity in children and adolescents.

Vitamin A is essential in human nutrition because they can prevent certain diseases. Food intake and lifestyle could affect the serum concentration of this nutrient, especially fruits and vegetables containing vitamin $\mathrm{A}$ and its precursors are low in intake (26). Studies had shown that obese individuals were deficient in vitamin A $(27,28)$. However, it has not been determined whether this deficiency is due to poor diet or other factors, such as oxidative stress and inflammatory processes caused by obesity (29). In addition, studies had shown that BMI was negatively correlated with the serum concentrations of $\beta$-carotene, $\beta$-cryptoxanthin, retinol, and other carotenoids ( $\alpha$-carotene and lutein + zeaxanthin) $(30,31)$. Although subjects with different BMI ranges had similar carotenoid intakes, these studies showed that obese subjects had lower serum levels of vitamin A precursors. At the same time, studies had confirmed that higher serum levels of $\alpha$-carotene, trans- $\beta$-carotene, and cis- $\beta$-carotene were associated with a lower risk of overweight or obesity in children, while an increase in retinol concentration was associated with an increased risk $(32,33)$. The mechanism that may lead to the discovery of an inverse association between serum carotenoids and obesity is the difference in dietary fruits, vegetables, and energy intake between obese and non-obese subjects (34). Although obese subjects may consume too much energy food, they may not be able to meet all their micronutrient needs. In addition, compared with lean people, obese/overweight individuals will deposit more b-carotene in their adipose tissue, so compared with lean people, the serum carotenoid concentration may be lower $(7,35)$.

Vitamin D deficiency is characterized by circulating 25-hydroxy vitamin D [25(OH)D] plasma levels below $20 \mathrm{ng} / \mathrm{mL}$, 


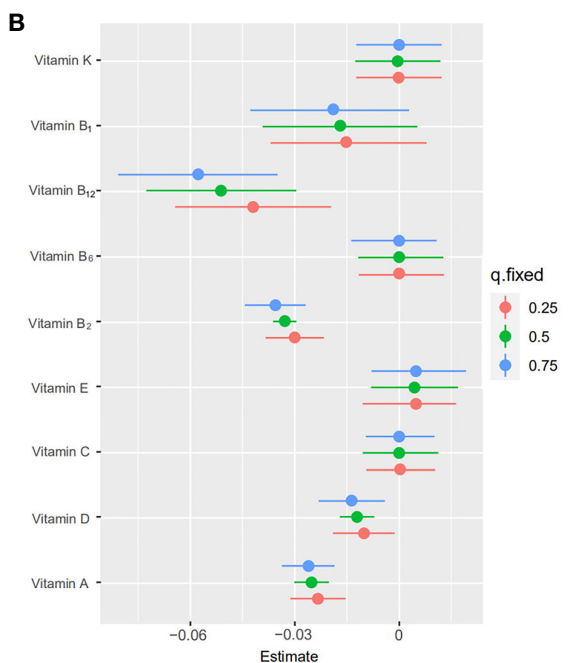

FIGURE 3 | Joint effect [95\% confidence intervals (CI)] of the five vitamins [vitamin $A$, vitamin $B_{1}$, vitamin $B_{2}$, vitamin $B_{6}$, vitamin $B_{12}$, vitamin $C$, vitamin $D$, vitamin $E$, vitamin $\mathrm{K}$ ] on obesity by using Bayesian kernel machine regression (BKMR) model. The results were adjusted for age, sex, education level, race/ethnicity, povertyincome ratio, serum cotinine and daily energy intake. (A) Overall effect of five vitamins (estimates and 95\%Cl). The figure plots the estimated change in a latent continuous outcome (continuous marker of obesity) when all the vitamins at fixed percentiles were compared to all the vitamins at their 50 th percentile. (B) Single vitamin association (estimates and $95 \% \mathrm{Cl}$, estimated zero means null). This plot compares a latent continuous outcome when a single vitamin is at the 75 th vs 25 th percentile, when all the other vitamins are fixed at either the 25th, 50th, or 75th percentile.

which is associated with some chronic diseases, such as insulin resistance, metabolic syndrome, atherosclerosis and obesity (36, 37). Since the skin is exposed to the sun, the endogenous production of vitamin $\mathrm{D}$ is the main source of this vitamin, because very few foods contain this vitamin, and the content is at very low levels (38). The possible reason for the low vitamin $\mathrm{D}$ status during obesity is that obese individuals have low vitamin $\mathrm{D}$ intake, less physical activity, resulting in less exposure to the sun, and reduced intestinal absorption of nutritional vitamin $\mathrm{D}$ by individuals undergoing bariatric surgery (39-41). People living in high latitudes were rarely exposed to the sun, and the relationship between the low rate of endogenous vitamin D synthesis and the high rate of obese and overweight individuals had been confirmed (42). Increased obesity in children and adolescents is associated with vitamin D deficiency (43). Children with insufficient vitamin D intake (daily intake $<70$ IU) have higher body weight, BMI, waist circumference, and waistto-hip (44). Contrary to other findings, adolescents showed the same relationship, but the concentration of parathyroid hormone did not increase (45). It seems that the relationship between vitamin $\mathrm{D}$ deficiency and obesity in adolescents is caused by metabolic factors in this age group, mainly due to hormonal changes $(46,47)$.

There is little evidence to support a direct causal relationship between vitamin $\mathrm{D}$ and obesity and metabolic parameters. Inflammation may be one of the mechanisms that explain the association between vitamin $\mathrm{D}$ and obesity and metabolic parameters (48). It has been reported that vitamin $\mathrm{D}$ can inhibit the concentration of $25(\mathrm{OH}) \mathrm{D}$ as an acute phase reactant in inflammation. Obesity is now widely regarded as a chronic, low-grade systemic inflammatory state $(49,50)$. It has been reported that the improvement of $25(\mathrm{OH}) \mathrm{D}$ concentration can enhance the beneficial effect of weight loss, which may be at least partly attributed to the anti-inflammatory effect of vitamin D. Although a meta-analysis of randomized clinical trials had shown that vitamin D supplementation had no effect on inflammatory biomarkers in overweight/obese adults, more research evidence is needed. Recently, studies had shown that vitamin $\mathrm{D}$ supplementation could reduce inflammation biomarkers [ie interleukin-6 (IL-6) levels] in adults with metabolic syndrome. More randomized clinical trials in obese children and adolescents are needed to clarify the mechanism $(51,52)$. At the same time, obesity-related vitamin $\mathrm{D}$ deficiency may be due to the reduced bioavailability of subcutaneous fat tissue and diet-derived vitamin $\mathrm{D}$, because it is deposited in various fat areas of the body. In addition, LC-MS/MS detected vitamin D3 in subcutaneous adipose tissue of obese subjects, and the results showed that the concentration of vitamin D3 in adipose tissue was more than 10 times higher than that in serum. These findings indicate that vitamin $\mathrm{D}$, as a fat-soluble vitamin, may accumulate and segregate in adipose tissue and cannot enter the circulation to produce $25(\mathrm{OH}) \mathrm{D}$ in the liver. This may lead to a decrease in plasma $25(\mathrm{OH}) \mathrm{D}$ levels in subjects with excessive accumulation of adipose tissue $(53,54)$.

Few comparable studies had investigated the relationship between children's vitamin B intake and physical obesity. A study reported that the intake of niacin in overweight boys was significantly lower than that of non-overweight boys (55). Studies had found that long-term use of vitamin B-6 and B-12 supplements is significantly associated with lower levels of weight gain in overweight or obese men and women $(56,57)$. These studies, combined with the findings of B-vitamins status and obesity, strengthen the argument that it may play an important role in adipogenesis. This association may be caused 


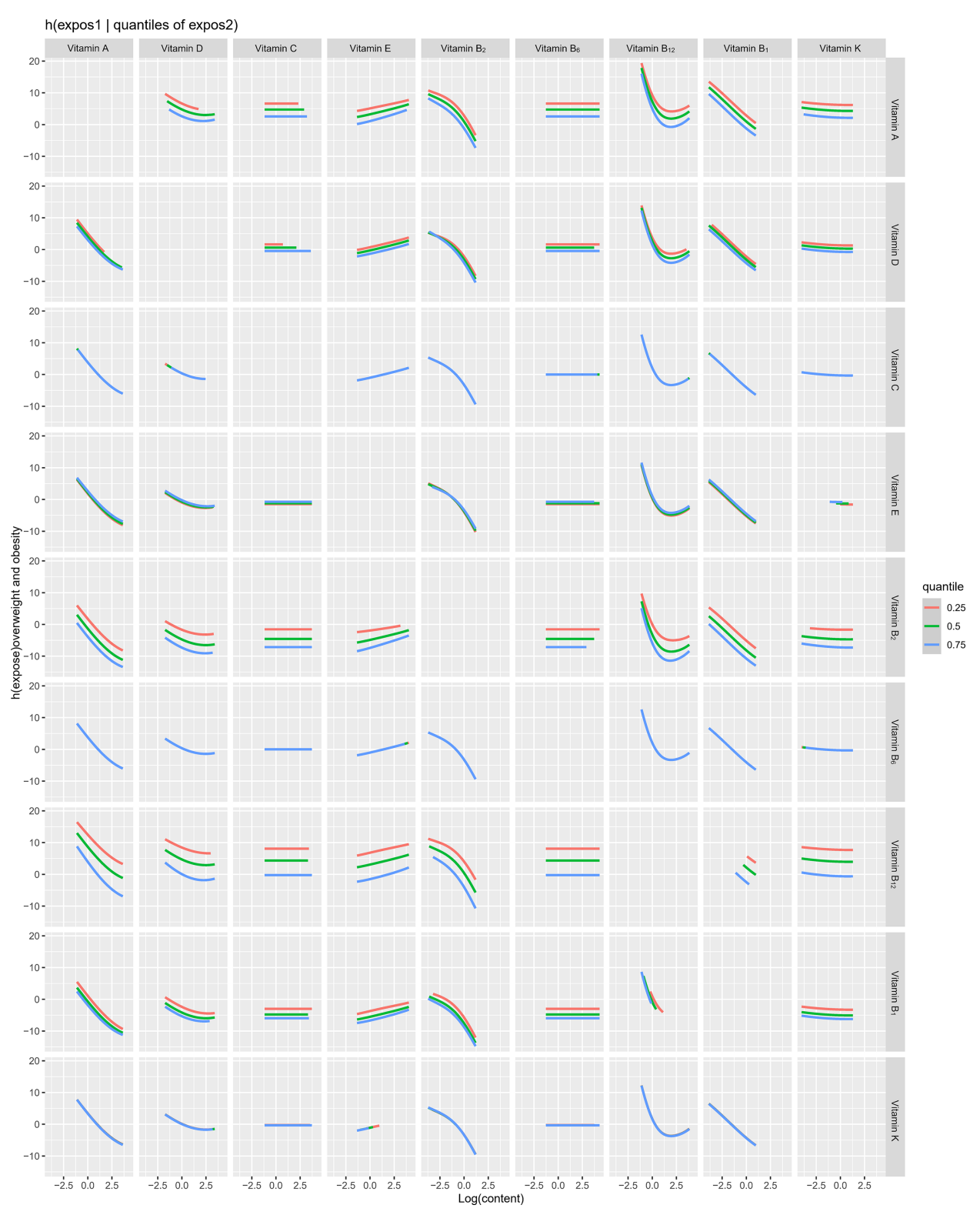

FIGURE 4 | Bi-variate exposure response functions of each two vitamins in obesity among children and adolescents.

by the inflammatory state found in obese individuals, which can lead to changes in the physiology of the B-vitamins (58). Obesity can induce systemic oxidative stress, which may lead to imbalance of adipocytokines and systemic inflammation, and subsequently reduces the serum concentration of vitamin B-6 (59). For example, A study had found that low-circulation 5'pyridoxal phosphate (PLP) was associated with an increase in inflammation marker C-reactive protein. It had also been found that there was a negative correlation between dietary intake of PLP or vitamin B-6 and coronary heart disease (60). These findings indicated that when there was an underlying inflammatory process, the use of vitamin B-6 could decrease the concentration of PLP in obesity and coronary heart disease.
A new BKMR analysis method was implemented to capture the reliable association between environmental multivitamins intake and obesity in children and adolescents. We found that a mixture of vitamin $A$, vitamin $B_{1}$, vitamin $B_{2}$, vitamin $B_{12}$, and vitamin $D$ had a significant positive combined effect on the risk of obesity in children. Therefore, any potential intervention for vitamin intake imbalances should specifically consider multivitamins instead of a single vitamin $(61,62)$. The BKMR results provided a non-linear analysis of the interaction between vitamins and a significant positive interaction between vitamin $B_{2}$ and vitamin $B_{12}$ was observed in this study. The mechanism underlying the observed interactions between B-vitamins and changes in BMI remains unknown. However, the current findings are biologically 
reasonable, because B-vitamins play an important role in the DNA methylation of obesity-related genes (63). In addition, vitamin $B_{2}$ and vitamin $B_{12}$ are the main determinants of one-carbon metabolism, among which methyl donors are formed. It had been studied that the co-expression of the Vitamin $\mathrm{B}_{2}$ and vitamin $B_{12}$ could increase the risk of obesity $(64,65)$.

The strengths of our research included a large sample size and sufficient statistical power to evaluate potential interactions. In addition, we used BKMR to explain the potential interaction between vitamin mixtures and obesity in children and adolescents. The most important limitation of the study was the cross-sectional design, which limited our ability to assess causality. In addition, we only evaluated the dietary intake of vitamins, which may overestimate the impact of vitamin content on obesity in children and adolescents. At last, a 24-hour recall may not be the best tool for assessing daily intake. For vitamins that vary greatly from day to day due to their distribution in different foods, this may introduce potential biases to the results.

\section{CONCLUSION}

We discover a combined negative effect of multivitamins on obesity in children and adolescents, and observe a significant interaction between vitamin B2 and vitamin B12. Further cohort studies are needed to clarify the health effects of multivitamins intake in larger population.

\section{DATA AVAILABILITY STATEMENT}

The original contributions presented in the study are included in the article/Supplementary Material. Further inquiries can be directed to the corresponding author.

\section{REFERENCES}

1. Kansra AR, Lakkunarajah S, Jay MS. Childhood and Adolescent Obesity: A Review. Front Pediatr (2020) 8:581461. doi: 10.3389/fped.2020.581461

2. Sluggett L, Wagner SL, Harris RL. Sleep Duration and Obesity in Children and Adolescents. Can J Diabetes (2019) 43:146-52. doi: 10.1016/j.jcjd. 2018.06.006

3. Styne DM, Arslanian SA, Connor EL, Farooqi IS, Murad MH, Silverstein JH, et al. Pediatric Obesity-Assessment, Treatment, and Prevention: An Endocrine Society Clinical Practice Guideline. J Clin Endocrinol Metab (2017) 102:709-57. doi: 10.1210/jc.2016-2573

4. Murer SB, Saarsalu S, Zimmermann J, Herter-Aeberli I. Risk Factors for Overweight and Obesity in Swiss Primary School Children: Results From a Representative National Survey. Eur J Nutr (2016) 55:621-9. doi: 10.1007/ s00394-015-0882-5

5. Petraroli M, Castellone E, Patianna V, Esposito S. Gut Microbiota and Obesity in Adults and Children: The State of the Art. Front Pediatr (2021) 9:657020. doi: 10.3389/fped.2021.657020

6. Azab SF, Saleh SH, Elsaeed WF, Elshafie MA, Sherief LM, Esh AM. Serum Trace Elements in Obese Egyptian Children: A Case-Control Study. Ital J Pediatr (2021) 40:20. doi: 10.1186/1824-7288-40-20

7. Gunanti IR, Marks GC, Al-Mamun A, Long KZ. Low Serum Vitamin B-12 and Folate Concentrations and Low Thiamin and Riboflavin Intakes are Inversely Associated With Greater Adiposity in Mexican American Children. J Nutr (2014) 144:2027-33. doi: 10.3945/jn.114.201202

\section{ETHICS STATEMENT}

The studies involving human participants were reviewed and approved by the National Center for Health Statistics Ethics Review Board. Written informed consent to participate in this study was provided by the participants' legal guardian/next of kin.

\section{AUTHOR CONTRIBUTIONS}

QC: design, analysis, drafting and revising manuscript. WT: design, analysis, drafting and revising manuscript. WZ: design, analysis and revising manuscript. MW: data collection and revising manuscript. All authors contributed to the article and approved the submitted version.

\section{FUNDING}

The project was supported in part by National Natural Science Foundation of China (NSFC 81803244) and Collaborative Innovation Program of Shanghai Municipal Health Commission (2020CXJQ01). The funders did not play any role in the study design, data collection and analysis, decision to publish or preparation of the manuscript.

\section{SUPPLEMENTARY MATERIAL}

The Supplementary Material for this article can be found online at: https://www.frontiersin.org/articles/10.3389/fendo.2021.816975/ full\#supplementary-material

8. Whitfield KC, da Silva L, Feldman F, Singh S, McCann A, McAnena L, et al Adequate Vitamin B and Riboflavin Status From Menus Alone in Residential Care Facilities in the Lower Mainland, British Columbia. Appl Physiol Nutr Metab (2019) 44:414-9. doi: 10.1139/apnm-2018-0459

9. Heyn GS, Corrêa LH, Magalhães KG. The Impact of Adipose Tissue-Derived miRNAs in Metabolic Syndrome, Obesity, and Cancer. Front Endocrinol (Lausanne) (2020) 11:563816. doi: 10.3389/fendo.2020.563816

10. Amengual J, Zhang N, Kemerer M, Maeda T, Palczewski K, Von Lintig J. STRA6 Is Critical for Cellular Vitamin A Uptake and Homeostasis. Hum Mol Genet (2014) 23:5402-17. doi: 10.1093/hmg/ddu258

11. Blaner WS. Vitamin A Signaling and Homeostasis in Obesity, Diabetes, and Metabolic Disorders. Pharmacol Ther (2019) 197:153-78. doi: 10.1016/ j.pharmthera.2019.01.006

12. Wu X, Ueland PM, Roper J, Koh GY, Liang X, Crott JW, et al. Combined Supplementation With Vitamin B-6 and Curcumin is Superior to Either Agent Alone in Suppressing Obesity-Promoted Colorectal Tumorigenesis in Mice. J Nutr (2021) 151:3678-88. doi: 10.1093/jn/nxab320

13. Karampela I, Sakelliou A, Vallianou N, Christodoulatos GS, Magkos F, Dalamaga M. Vitamin D and Obesity: Current Evidence and Controversies. Curr Obes Rep (2021) 10:162-80. doi: 10.1007/s13679-021-00433-1

14. Ellulu MS. Obesity, Cardiovascular Disease, and Role of Vitamin C on Inflammation: A Review of Facts and Underlying Mechanisms. Inflammopharmacology (2017) 25:313-28. doi: 10.1007/s10787-017-0314-7

15. Aruoma OI, Hausman-Cohen S, Pizano J, Schmidt MA, Minich DM, Joffe Y, et al. Personalized Nutrition: Translating the Science of NutriGenomics Into 
Practice: Proceedings From the 2018 American College of Nutrition Meeting. J Am Coll Nutr (2019) 38:287-301. doi: 10.1080/07315724.2019.1582980

16. Guo S, Niu J, Xv J, Fang B, Zhang Z, Zhao D, et al. Interactive Effects of Vitamins A and K on Laying Performance, Egg Quality, Tibia Attributes and Antioxidative Status of Aged Roman Pink Laying Hens. Animal (2021) 15:100242. doi: 10.1016/j.animal.2021.100242

17. Bobb JF, Valeri L, Claus Henn B, Christiani DC, Wright RO, Mazumdar M, et al. Bayesian Kernel Machine Regression for Estimating the Health Effects of Multi-Pollutant Mixtures. Biostatistics (2015) 16:493-508. doi: 10.1093/ biostatistics $/ \mathrm{kxu} 058$

18. Valeri L, Mazumdar MM, Bobb JF, Claus Henn B, Rodrigues E, Sharif OIA, et al. The Joint Effect of Prenatal Exposure to Metal Mixtures on Neurodevelopmental Outcomes at 20-40 Months of Age: Evidence From Rural Bangladesh. Environ Health Perspect (2017) 125:067015. doi: 10.1289/ EHP614

19. Johnson CL, Dohrmann SM, Burt VL, Mohadjer LK. National Health and Nutrition Examination Survey: Sample Design, 2011-2014. Vital Health Stat 2 (2014) 162:1-33.

20. Peeri NC, Egan KM, Chai W, Tao MH. Association of Magnesium Intake and Vitamin D Status With Cognitive Function in Older Adults: An Analysis of US National Health and Nutrition Examination Survey (NHANES) 2011 to 2014. Eur J Nutr (2021) 60:465-74. doi: 10.1007/s00394-020-02267-4

21. Clinical Guidelines on the Identification, Evaluation, and Treatment of Overweight and Obesity in Adults-The Evidence Report. National Institutes of Health. Obes Res (1998) Suppl 2:51S-209S. Avaliable from: https://xs. dailyheadlines.cc

22. Hunt ET, Brazendale K, Dunn C, Liu J, Hardin J, Beets MW, et al. Income, Race and Its Association With Obesogenic Behaviors of U.S. Children and Adolescents, NHANES 2003-2006. J Community Health (2019) 44:507-18. doi: 10.1007/s10900-018-00613-6

23. Skinner AC, Perrin EM, Moss LA, Skelton JA. Cardiometabolic Risks and Severity of Obesity in Children and Young Adults. N Engl J Med (2015) 373:1307-17. doi: 10.1056/NEJMoa1502821

24. Cai H, Huang J, Xu G, Yang Z, Liu M, Mi Y, et al. Prevalence and Determinants of Metabolic Syndrome Among Women in Chinese Rural Areas. PloS One (2012) 7:e36936. doi: 10.1371/journal.pone.0036936

25. Bobb JF. Bkmr: Bayesian Kernel Machine Regression, Version 0.2.0 (2017). Available at: https://cran.r-project.org/web/packages/bkmr/index.html.

26. Neuhouser ML, Rock CL, Eldridge AL, Kristal AR, Patterson RE, Cooper DA, et al. Serum Concentrations of Retinol, Alpha-Tocopherol and the Carotenoids Are Influenced by Diet, Race and Obesity in a Sample of Healthy Adolescents. J Nutr (2001) 131:2184-91. doi: 10.1093/jn/131.8.2184

27. Bermudez OI, Tucker KL. Trends in Dietary Patterns of Latin American Populations. Cad Saude Publica (2003) 19(Suppl 1):S87-99. doi: 10.1590/ S0102-311X2003000700010

28. Wei X, Peng R, Cao J, Kang Y, Qu P, Liu Y. Serum Vitamin A Status Is Associated With Obesity and the Metabolic Syndrome Among School-Age Children in Chongqing, China. Asia Pac J Clin Nutr (2016) 25:563-70. doi: 10.6133/apjcn.092015.03

29. Bento C, Matos A, Cordeiro A, Ramalho A. Serum Concentration of Vitamin A and Its Relationship With Body Adiposity, Oxidative Stress, and Cardiovascular Risk in Women With Recommended Dietary Intake of Vitamin A. Nutr Hosp (2020) 37:1135-42. doi: 10.20960/nh.03129

30. Botella-Carretero JI, Balsa JA, Vázquez C, Peromingo R, Díaz-Enriquez M, Escobar-Morreale HF. Retinol and Alpha-Tocopherol in Morbid Obesity and Nonalcoholic Fatty Liver Disease. Obes Surg (2010) 20:69-76. doi: 10.1007/ s11695-008-9686-5

31. de Souza Valente da Silva L, Valeria da Veiga G, Ramalho RA. Association of Serum Concentrations of Retinol and Carotenoids With Overweight in Children and Adolescents. Nutrition (2007) 23:392-7. doi: 10.1016/ j.nut.2007.02.009

32. Farook VS, Reddivari L, Mummidi S, Puppala S, Arya R, Lopez-Alvarenga JC. Genetics of Serum Carotenoid Concentrations and Their Correlation With Obesity-Related Traits in Mexican American Children. Am J Clin Nutr (2017) 106:52-8. doi: 10.3945/ajcn.116.144006

33. Albuquerque MN, Diniz Ada S, Arruda IK. Elevated Serum Retinol and Low Beta-Carotene But Not Alpha-Tocopherol Concentrations Are Associated
With Dyslipidemia in Brazilian Adolescents. J Nutr Sci Vitaminol (Tokyo) (2016) 62:73-80. doi: 10.3177/jnsv.62.73

34. Pratt KJ, Hill EB, Kiser HM, VanFossen CE, Braun A, Taylor CA, et al. Changes in Parent and Child Skin Carotenoids, Weight, and Dietary Behaviors Over Parental Weight Management. Nutrients (2021) 13:2227. doi: $10.3390 /$ nu13072227

35. Switzer BR, Atwood JR, Stark AH, Hatch JW, Travis R, Ullrich F, et al. Plasma Carotenoid and Vitamins a and E Concentrations in Older African American Women After Wheat Bran Supplementation: Effects of Age, Body Mass and Smoking History. J Am Coll Nutr (2005) 24(3):217-26. doi: 10.1080/ 07315724.2005.10719468

36. Holick MF. Vitamin D Status: Measurement, Interpretation, and Clinical Application. Ann Epidemiol (2009) 19:73-88. doi: 10.1016/j.annepidem. 2007.12.001

37. Aarts E, van Groningen L, Horst R, Telting D, van Sorge A, Janssen I, et al. Vitamin D Absorption: Consequences of Gastric Bypass Surgery. Eur J Endocrinol (2011) 164:827-32. doi: 10.1530/EJE-10-1126

38. Midtbø LK, Nygaard LB, Markhus MW, Kjellevold M, Lie $\varnothing$, Dahl L, et al. Vitamin D Status in Preschool Children and Its Relations to Vitamin D Sources and Body Mass Index-Fish Intervention Studies-KIDS (FINS-KIDS). Nutrition (2020) 70:110595. doi: 10.1016/j.nut.2019.110595

39. Vranić L, Mikolašević I, Milić S. Vitamin D Deficiency: Consequence or Cause of Obesity? Medicina (Kaunas) (2019) 55(9):541. doi: 10.3390/ medicina55090541

40. Savastano S, Barrea L, Savanelli MC, Nappi F, Di Somma C, Orio F, et al. Low Vitamin D Status and Obesity: Role of Nutritionist. Rev Endocr Metab Disord (2017) 18(2):215-25. doi: 10.1007/s11154-017-9410-7

41. Sergeev IN. Vitamin D Status and Vitamin D-Dependent Apoptosis in Obesity. Nutrients (2020) 12(5):1392. doi: 10.3390/nu12051392

42. Dix CF, Barcla JL, Wright ORL. The Role of Vitamin D in Adipogenesis. Nutr Rev (2018) 76:47-59. doi: 10.1093/nutrit/nux056

43. Fiamenghi VI, Mello ED. Vitamin D Deficiency in Children and Adolescents With Obesity: A Meta-Analysis. J Pediatr (Rio J) (2021) 97:273-9. doi: 10.1016/j.jped.2020.08.006

44. Rojas LZ, Quintero-Lesmes DC, Gamboa-Delgado EM, Guio E, Serrano NC. Prevalence of Vitamin D Status and Its Association With Overweight or Obesity in a Population of Colombian Children and Adolescents. J Nutr Sci (2020) 9:e55. doi: 10.1017/jns.2020.47

45. Durá-Travé T, Gallinas-Victoriano F, Chueca-Guindulain MJ, Berrade-Zubiri S, Urretavizcaya-Martinez M, Ahmed-Mohamed L. Assessment of Vitamin D Status and Parathyroid Hormone During a Combined Intervention for the Treatment of Childhood Obesity. Nutr Diabetes (2019) 9:18. doi: 10.1038/ s41387-019-0083-z

46. Pereira-Santos M, Costa PR, Assis AM, Santos CA, Santos DB. Obesity and Vitamin D Deficiency: A Systematic Review and Meta-Analysis. Obes Rev (2015) 16(4):341-9. doi: 10.1111/obr.12239

47. Plesner JL, Dahl M, Fonvig CE, Nielsen TRH, Kloppenborg JT, Pedersen O, et al. Obesity is Associated With Vitamin D Deficiency in Danish Children and Adolescents. J Pediatr Endocrinol Metab (2018) 31(1):53-61. doi: 10.1515/jpem-2017-0246

48. Waldron JL, Ashby HL, Cornes MP, Bechervaise J, Razavi C, Thomas OL. Vitamin D: A Negative Acute Phase Reactant. J Clin Pathol (2013) 66:620-2. doi: 10.1136/jclinpath-2012-201301

49. Villarroya J, Cereijo R, Villarroya F. An Endocrine Role for Brown Adipose Tissue? Am J Physiol Endocrinol Metab (2013) 305:E567-72. doi: 10.1152/ ajpendo.00250.2013

50. Zhang Y, Proenca R, Maffei M, Barone M, Leopold L, Friedman JM. Positional Cloning of the Mouse Obese Gene and Its Human Homologue. Nature (1994) 372:425-32. doi: 10.1038/372425a0

51. Zittermann A, Frisch S, Berthold HK, Götting C, Kuhn J, Kleesiek K, et al. Vitamin D Supplementation Enhances the Beneficial Effects of Weight Loss on Cardiovascular Disease Risk Markers. Am J Clin Nutr (2009) 89:1321-7. doi: 10.3945/ajen.2008.27004

52. Ghashut RA, Talwar D, Kinsella J, Duncan A, McMillan DC. The Effect of the Systemic Inflammatory Response on Plasma Vitamin $25(\mathrm{OH})$ D Concentrations Adjusted for Albumin. PloS One (2014) 9:e92614. doi: 10.1371/journal.pone.0092614 
53. Zuk A, Fitzpatrick T, Rosella LC. Effect of Vitamin D3 Supplementation on Inflammatory Markers and Glycemic Measures Among Overweight or Obese Adults: A Systematic Review of Randomized Controlled Trials. PloS One (2016) 11:e0154215. doi: 10.1371/journal.pone.0154215

54. Salekzamani S, Bavil AS, Mehralizadeh H, Jafarabadi MA, Ghezel A, Gargari BP. The Effects of Vitamin D Supplementation on Proatherogenic Inflammatory Markers and Carotid Intima Media Thickness in Subjects With Metabolic Syndrome: A Randomized Double-Blind PlaceboControlled Clinical Trial. Endocrine (2017) 57:51-9. doi: 10.1007/s12020017-1317-2

55. Zhou SS, Li D, Zhou YM, Sun WP, Liu QG. B-Vitamin Consumption and the Prevalence of Diabetes and Obesity Among the US Adults: Population Based Ecological Study. BMC Public Health (2010) 10:746. doi: 10.1186/1471-245810-746

56. Carrodeguas L, Kaidar-Person O, Szomstein S, Antozzi P, Rosenthal R. Preoperative Thiamine Deficiency in Obese Population Undergoing Laparoscopic Bariatric Surgery. Surg Obes Relat Dis (2005) 1(6):517-22. doi: 10.1016/j.soard.2005.08.003

57. Kardaş F, Yücel AD, Kendirci M, Kurtoğlu S, Hatipoğlu N, Akın L, et al. Evaluation of Micronutrient Levels in Children and Adolescents With Obesity and Their Correlation With the Components of Metabolic Syndrome. Turk J Pediatr (2021) 63:48-58. doi: 10.24953/turkjped.2021.01.006

58. Christensen MHE, Fadnes DJ, Røst TH, Pedersen ER, Andersen JR, Våge V, et al. Inflammatory Markers, the Tryptophan-Kynurenine Pathway, and Vitamin B Status After Bariatric Surgery. PloS One (2018) 13:e0192169. doi: 10.1371/journal.pone.0192169

59. Lira FS, Rosa JC, Cunha CA, Ribeiro EB, do Nascimento CO, Oyama LM. Supplementing Alpha-Tocopherol (Vitamin E) and Vitamin D3 in High Fat Diet Decrease IL-6 Production in Murine Epididymal Adipose Tissue and 3T3-L1 Adipocytes Following LPS Stimulation. Lipids Health Dis (2011) 10:37. doi: 10.1186/1476-511X-10-37

60. Sakakeeny L, Roubenoff R, Obin M, Fontes JD, Benjamin EJ, Bujanover Y. Plasma Pyridoxal-5-Phosphate is Inversely Associated With Systemic Markers of Inflammation in a Population of U.S. Adults. J Nutr (2012) 142(7):1280-5. doi: $10.3945 /$ jn.111.153056
61. Gariballa S, Afandi B, Abuhaltem M, Yassin J, Habib H, Ibrahim W. Oxidative Damage and Inflammation in Obese Diabetic Emirati Subjects Supplemented With Antioxidants and B-Vitamins: A Randomized Placebo-Controlled Trail. Nutr Metab (Lond) (2013) 10(1):21. doi: 10.1186/1743-7075-10-21

62. Friso S, Choi SW. Gene-Nutrient Interactions and DNA Methylation. J Nutr (2002) 132(8 Suppl):2382S-7S. doi: 10.1093/jn/132.8.2382S

63. Park HJ, Bailey LB, Shade DC, Hausman DB, Hohos NM, Meagher RB. Distinctions in Gene-Specific Changes in DNA Methylation in Response to Folic Acid Supplementation Between Women With Normal Weight and Obesity. Obes Res Clin Pract (2017) 11:665-76. doi: 10.1016/j.orcp.2017. 06.004

64. Bailey LB. Folate and Vitamin B12 Recommended Intakes and Status in the United States. Nutr Rev (2004) 62(6 Pt 2):S14-20. doi: 10.1111/j.17534887.2004.tb00065.x

65. Relton CL, Davey Smith G. Epigenetic Epidemiology of Common Complex Disease: Prospects for Prediction, Prevention, and Treatment. PloS Med (2010) 7(10):e1000356. doi: 10.1371/journal.pmed.1000356

Conflict of Interest: The authors declare that the research was conducted in the absence of any commercial or financial relationships that could be construed as a potential conflict of interest.

Publisher's Note: All claims expressed in this article are solely those of the authors and do not necessarily represent those of their affiliated organizations, or those of the publisher, the editors and the reviewers. Any product that may be evaluated in this article, or claim that may be made by its manufacturer, is not guaranteed or endorsed by the publisher.

Copyright (๑) 2022 Tang, Zhan, Wei and Chen. This is an open-access article distributed under the terms of the Creative Commons Attribution License (CC BY). The use, distribution or reproduction in other forums is permitted, provided the original author(s) and the copyright owner(s) are credited and that the original publication in this journal is cited, in accordance with accepted academic practice. No use, distribution or reproduction is permitted which does not comply with these terms. 